\title{
CLOSE-TO-CONVEX MULTIVALENT FUNCTIONS WITH RESPECT TO WEAKLY STARLIKE FUNCTIONS
}

\author{
BY \\ DAVID STYER ( 1 )
}

\begin{abstract}
It is the object of this article to define close-to-convex multivalent functions in terms of weakly starlike multivalent functions. Six classes are defined, and shown to be equal. These generalize the class of close-to-convex functions developed by Livingston in the article, p-valent close-to-convex functions, Trans. Amer. Math. Soc. 115 (1965), $161-179$.
\end{abstract}

1. In this paper we consider several ways to define multivalent close-to-convex functions with respect to weakly starlike functions, and we study the relationships between the classes defined. The theory is based on J. A. Hummel's paper [1] on $p$-valent weakly starlike functions, and the paper by $A$. E. Livingston [3] on $p$-valent close-to-convex functions.

This section is devoted to the definitions and theorems which we will use.

Definition 1. Let $S_{0}(1)$ be the class of functions $f$ univalent in $U=$ $\{z:|z|<1\}$ such that $f(0)=0$ and $f(U)$ is starshaped with respect to $0 . S_{0}(1)$ is the class of starlike univalent functions.

Note that we do not insist that $f^{\prime}(0)=1$. It is well known that

$f \in S_{0}(1)$ if and only if $f$ is regular in $U$, has one zero there (counting multiplicity) and $\operatorname{Re}\left(z f^{\prime}(z) / f(z)\right)>0$ for all $z \in U$.

Definition 2. Let $S_{a}(p)$ be the class of functions $f$ regular in $U$, with $p$ zeros there (counting multiplicity) and such that $\operatorname{Re}\left(z f^{\prime}(z) / f(z)\right)>0$ for all $z$ in some annulus $A_{\rho}=\{z: \rho<|z|<1\}$.

These are the "standard" multivalent starlike functions. The extension to the class of weakly starlike functions, as developed by Hummel [1], is direct:

Definition 3. Let $S_{w}(p)$ be the class of functions $f$ regular in $U$, with $p$ zeros there and such that

$$
\liminf _{r \rightarrow 1-}\left[\min _{|z|=r} \operatorname{Re} \frac{z f^{\prime}(z)}{f(z)}\right] \geq 0
$$

Received by the editors June 29, 1971.

AMS 1970 subject classifications. Primary 30A32.

Key words and phrases. Multivalent functions, close-to-convex functions, weakly starlike functions.

(1) This paper was written while the author was a Charles Phelps Taft Postdoctoral Fellow at the University of Cincinnati. 
Definition 4. We will say that the sequence $\left\{f_{n}\right\}$ of functions in $U$ converges almost uniformly to $f$ if $\left\{f_{n}\right\}$ converges uniformly to $f$ on each compact subset of $U$.

The following results on weakly starlike functions may be found in Hummel [1].

Proposition 1. $f \in S_{w}(p)$ if and only if $f$ has $p$ zeros and $f$ is the almost uniform limit of a sequence of functions in $S_{a}(p)$.

Since functions in $S_{a}(p)$ are $p$-valent, this shows that functions in $S_{w}(p)$ are also $p$-valent. It also shows that $f^{\prime}$ has at most $p-1$ zeros.

Let $\Psi\left(z, z_{0}\right)=\left(z-z_{0}\right)\left(1-\bar{z}_{0} z\right) / z, \Psi(z, 0) \equiv 1$.

Proposition 2. $f \in S_{w}(p)$ if and only if there is a function $b \in S_{0}(1)$ such that

$$
f(z)=[b(z)]^{p} \prod_{i=1}^{p} \Psi\left(z, z_{i}\right), \quad\left|z_{i}\right|<1 .
$$

Here each $z_{i}$ is a zero of $f$.

We follow Livingston in our definition of close-to-convex functions.

Definition 5. Let $K(p)$ be the class of functions $F$, regular in $U$, with $F(0)=$ 0 , such that there is a function $f \in S_{a}(p)$ for which $f(0)=0$ and

$$
\operatorname{Re}\left[z F^{\prime}(z) / f(z)\right]>0 \text { in some annulus } A_{\rho}=\{z: \rho<|z|<1\} .
$$

We note that Livingston singles out the subclass for which $F$ and $f$ are both regular on $C_{1}=\{z:|z|=1\}$ and satisfy (3) on $C_{1}$.

The class $K(1)$ is automatically reduced to close-to-convex univalent functions of Kaplan [2]:

$H \in K(1)$ if and only if there is a function $b \in S_{0}(1)$ such that

$$
\operatorname{Re}\left[z H^{\prime}(z) / b(z)\right]>0 \text { for all } z \in U \text {. }
$$

Proposition 3. Let $F \in K(p)$. Then $F$ is at most p-valent in $U$, and $F^{\prime}$ bas exactly $p-1$ zeros in $U$.

The proof of this proposition may be found in Livingston [3]. It depends on a lemma by Umezawa [5].

2. Weakly close-to-convex functions. In this section we define two classes of functions which both extend the class $K(p)$.

Definition A. Let $F$ be regular and nonconstant in $U(p \geq 1)$. F belongs to the class $K_{W A}(p)$ if $F(0)=0$ and there is a function $f \in S_{w}(p), f(0)=0$, such that 


$$
\liminf _{r \rightarrow 1-}\left[\min _{|z|=r} \operatorname{Re} \frac{z F^{\prime}(z)}{f(z)}\right] \geq 0
$$

Definition B. $F$ belongs to the class $K_{W B}(p)$ if there are $F_{n} \in K(p)$ and $f_{n} \in S_{a}(p), f_{n}(0)=0, n=1,2, \cdots$, such that

$$
\begin{aligned}
& F_{n} \rightarrow F \text { almost uniformly in } U \text { and } F \not 0, \\
& f_{n} \rightarrow f \text { almost uniformly in } U \text { and } f \in S_{w}(p),
\end{aligned}
$$

and

$$
\operatorname{Re}\left\{z F_{n}^{\prime}(z) / f_{n}(z)\right\}>0 \text { for } \rho_{n}<|z|<1, n=1,2, \cdots
$$

Remark. If the $F_{n}, f_{n}, n=1,2, \cdots$, were given the added condition that they all be regular on $C_{1}$ and if (6) were changed to be true on $z \in C_{1}$, then the same class of functions would result. This is because we may choose a sequence $\left\{r_{n}\right\}$ such that $r_{n} \rightarrow 1, F_{n}\left(r_{n} z\right) \in K(p), f_{n}\left(r_{n} z\right) \in S_{a}(p)$.

Both of these classes are obvious extensions of $K(p)$. We prove

Proposition 4. $K(p) \subseteq K_{W A}(p) \subseteq K_{W B}(p)$.

Proof. We only need show that $K_{W A}(p) \subseteq K_{W B}(p)$.

Let $F \in K_{W A}(p)$ and $f \in S_{w}(p), f(0)=0$, satisfy (5). Suppose $f(z)=$ $[b(z)]^{p} \Pi \Psi\left(z, z_{i}\right)$ as in Proposition 2. Let

$$
H_{t}(z)=\int_{0}^{z}\left[\frac{b(t z)}{b(z)}\right]^{p}\left(F^{\prime}(z)+(1-t) \frac{f(z)}{z}\right) d z
$$

where $0<t<1$. That $f_{t}(z)=[b(t z)]^{p} \Pi \Psi\left(z, z_{i}\right)$ is in $S_{a}(p)$ is shown in Hummel [1]. A little manipulation shows that $z H_{t}^{\prime}(z) / f_{t}(z)=z F^{\prime}(z) / f(z)+1-t$. Thus

$$
\liminf _{r \rightarrow 1-}\left[\min _{|z|=r} \operatorname{Re} \frac{z H_{t}^{\prime}(z)}{f_{t}(z)}\right]=\liminf _{r \rightarrow 1-}\left[\min _{|z|=r} \operatorname{Re} \frac{z F^{\prime}(z)}{f(z)}\right]+1-t \geq 1-t>0 .
$$

Therefore, in some annulus, $\rho_{t}<|z|<1, \operatorname{Re}\left(z H_{t}^{\prime}(z) / f_{t}(z)\right)>0$.

$f_{t} \rightarrow f$ almost uniformly on $U$ as $t \rightarrow 1$ (see Hummel [1]).

We will show that $H_{t} \rightarrow F$ almost uniformly on $U$ as $t \rightarrow 1$. This will complete the proof that $F \in K_{W B}(p)$.

Let $|z| \leq r<1$. Then

$$
\left|H_{t}(z)-F(z)\right| \leq \int\left|\left[\frac{b(t z)}{b(z)}\right]^{p}\left(F^{\prime}(z)+(1-t) \frac{f(z)}{z}\right)-F^{\prime}(z)\right||d z|
$$

where the path of integration is the line segment from 0 to $z$. But the quantity under the integral is 


$$
\left|F^{\prime}(z)\left(\left[\frac{b(t z)}{b(z)}\right]^{p}-1\right)+(1-t) \frac{f(z)}{z}\left[\frac{b(t z)}{b(z)}\right]^{p}\right|
$$

which can be made arbitrarily small for $t$ near 1 while $|z| \leq r$. Therefore, $H_{t} \rightarrow F$ almost uniformly as $t \rightarrow 1$. This completes the proof.

3. More weakly starlike functions. In this section we define a class of functions which is a slight extension of $S_{w}(p)$. We then show that functions close-toconvex with respect to this larger class have some simple and useful properties.

Definition 6. Let $S_{w c}(p)$ be the class of functions $f$ of the form

$$
f(z)=[b(z)]^{p} \prod_{i=1}^{p} \Psi\left(z, z_{i}\right), \quad\left|z_{i}\right| \leq 1,1 \leq i \leq p,
$$

and $b \in S_{0}(1)$. Let $S_{w c}(0)$ be the set of nonzero constant functions.

The only difference between (7) and (2) is that in (7) we may have $\left|z_{i}\right|=1$. This is significant in terms of being "starlike" with respect to zero. A function in $S_{w c}(p)$ may have no zero in $U$. Nevertheless, the author shows in another article [4] that most of the theory of the class $S_{w}(p)$ carries over to $S_{w c}(p)$.

We will use the uniform convergence property of $S_{w c}(p)$. Namely,

Proposition 5. $f \in S_{w c}(p)$ if and only if $f \neq 0$ and $f$ is the almost uniform limit of a sequence of functions in $S_{a}(p)$.

This is much the same as Proposition 1, and a proof need not be included.

Definition C. A function $F \neq 0$ belongs to the class $K_{W C}(p)$ if there are $F_{n} \in K(p), n=1,2, \cdots$, such that $F_{n} \rightarrow F$ almost uniformly in $U$.

Note that here we may assume there are $f_{n} \in S_{a}(p), f_{n}(0)=0, n=1,2, \cdots$, such that $f_{n} \rightarrow f$ almost uniformly in $U, f \in S_{w c}(p)$, and

$$
\operatorname{Re}\left\{z F_{n}^{\prime}(z) / f_{n}(z)\right\}>0, \quad \rho_{n}<|z|<1, n=1,2, \cdots .
$$

Thus $K_{W C}(p)$ is trivially an extension of $K_{W B}(p)$.

Definition D. A function $F$ belongs to the class $K_{W D}(p)$ if there is a function $H \in K(1)$ and a function $g \in S_{w c}(p-1)$ such that

$$
F(z)=\int_{0}^{z} g(z) H^{\prime}(z) d z .
$$

Proposition 6. $K_{W B}(p) \subseteq K_{W C}(p) \subseteq K_{W D}(p)$.

Proof. We only need show that $K_{W C}(p) \subseteq K_{W D}(p)$.

Let $F \in K_{W C}(p)$ and suppose $F_{n}$ and $f_{n^{\prime}}, n=1,2, \cdots$, are as defined in Definition $C$. We may then assume without loss of generality that the $F_{n}$ and the $f_{n}$ are regular on $C_{1}$ and $\operatorname{Re}\left\{z F_{n}^{\prime}(z) / f_{n}(z)\right\}>0$ on $C_{1}$.

Let $f_{n}(z)=\left[b_{n}(z)\right]^{p} \Pi \Psi\left(z, z_{i}\right)$. Suppose that the zeros of $F_{n}^{\prime}$ are $\alpha_{1}^{(n)}, \cdots, \alpha_{p-1}^{(n)}$. 
Then

$$
\operatorname{Re}\left\{\frac{z F_{n}^{\prime}(z)\left[\Pi \Psi\left(z, \alpha_{i}^{(n)}\right)\right]^{-1}}{\left[b_{n}(z)\right]^{p}}\right\}>0 \text { on } C_{1} .
$$

But this is the real part of a regular function, so it is positive throughout $U$. Also, $H_{n}^{\prime}(z)=F_{n}^{\prime}(z) /\left(\left[b_{n}(z)\right]^{p-1} \Pi \Psi\left(z, \alpha_{i}^{(n}\right)\right)$ is regular in $U$, and $\operatorname{Re}\left\{z H_{n}^{\prime}(z) / h_{n}(z)\right\}>0$ in $|z| \leq 1$.

Now $b_{n} \rightarrow b$ almost uniformly in $U$, and $b \in S_{0}(1)$.

$$
H_{n}^{\prime}(z) \rightarrow \frac{F^{\prime}(z)}{[b(z)]^{p-1} \Pi \Psi\left(z, \alpha_{i}\right)} \text { almost uniformly in } U,
$$

where $a_{i}^{(n)} \rightarrow a_{i}, 1 \leq i \leq p-1$. Let $H^{\prime}(z)=F^{\prime}(z) /\left([b(z)]^{p-1} \Pi \Psi\left(z, \alpha_{i}\right)\right)$. Thus $H^{\prime}$ is not identically zero. Furthermore, $z H_{n}^{\prime}(z) / b_{n}(z) \rightarrow z H^{\prime}(z) / b(z)$ almost uniformly on $U$, so $\operatorname{Re}\left\{z H^{\prime}(z) / b(z)\right\} \geq 0$ for all $z \in U$. For $H(z)=\int_{0}^{z} H^{\prime}(z) d z$, $H \in K(1)$. That is, if $z H^{\prime}(z) / b(z)$ is not constant, then $\operatorname{Re}\left\{z H^{\prime}(z) / b(z)\right\}>0$ for all $z \in U$. If $z H^{\prime}(z) / b(z)=i a$ for some $a \in \mathbf{R}$, then $i a b(z) \in S_{0}(1)$ and $z H^{\prime}(z) / i a b(z)=1$, which has positive real part.

Let $g(z)=[b(z)]^{p-1} \Pi \Psi\left(z, \alpha_{i}\right)$. Thus $F^{\prime}(z)=g(z) H^{\prime}(z)$, where $H \in K(1)$ and $g \in S_{w c}(p-1), F \in K_{W D}(p)$. This completes the proof.

Definition E. Let $F$ be regular in $U$, with $F(0)=0$. $F$ belongs to the class $K_{W E}(p)$ if there is a function $f \in S_{w c}(p)$ such that $f(0)=0$ and

$$
\operatorname{Re}\left\{z F^{\prime}(z) / f(z)\right\}>0 \text { for all } z \in U \text {. }
$$

Proposition 7. $K_{W D}(p) \subseteq K_{W E}(p)$.

Proof. Let $F \in K_{W D}(p)$. Then there is an $H \in K(1)$ and a $g \in S_{w c}(p-1)$ such that $F^{\prime}(z)=g(z) H^{\prime}(z)$. Also there is a function $b \in S_{0}(1)$ such that $\operatorname{Re}\left\{z H^{\prime}(z) / b(z)\right\}>0$ in $U$. Thus

$$
\operatorname{Re}\left\{z F^{\prime}(z) / g(z) b(z)\right\}=\operatorname{Re}\left\{z g(z) H^{\prime}(z) /^{\prime} g(z) b(z)\right\}>0 \text { in } U .
$$

Now $g(z)=\left[g_{1}(z)\right]^{p-1} \Pi \Psi\left(z, z_{i}\right)$, where $g_{1} \in S_{0}(1)$, so $b(z)\left[g_{1}(z)\right]^{p-1}=$ $\left[f_{1}(z)\right]^{p}$ for some $f_{1} \in S_{0}(1)$. Thus $g(z) b(z)=\left[f_{1}(z)\right]^{p} \Pi \Psi\left(z, z_{i}\right) \equiv f(z)$. Hence $\operatorname{Re}\left\{z F^{\prime}(z) / f(z)\right\}>0$ in $U$, and $f \in S_{w c}(p), f(0)=0, F \in K_{W E}(p)$. This completes the proof.

4. A subclass of $K_{W A}(p)$. In this section we unify our results. First it is desirable to define a final class of close-to-convex functions.

Definition $O$. Let $F$ be regular and nonconstant in $U . F$ belongs to the class $K_{W O}(p)$ if $F(0)=0$ and there is a function $b \in S_{0}(1)$ such that

$$
\liminf _{r \rightarrow 1-}\left[\min _{\lfloor z \mid=r} \operatorname{Re}\left\{\frac{z F^{\prime}(z)}{[b(z)]^{p}}\right\}\right] \geq 0
$$


It is completely trivial that $K_{W O}(p) \subseteq K_{W A}(p)$. It is less trivial, but true, that $K_{W E}(p) \subseteq K_{W O}(p)$. When we show this we will have

Theorem 1. Let $p$ be a positive integer. Then $K_{W A}(p)=K_{W B}(p)=K_{W C}(p)=$ $K_{W D}(p)=K_{W E}(p)=K_{W O}(p)$. If we let $K_{W}(p)$ stand for this class, then any function $F \in K_{W}(p)$ is at most p-valent and $F^{\prime}$ bas at most $p-1$ zeros in $U$. Furthermore, $K(p) \subseteq K_{W}(p)$ when $p>1$.

That a function $F \in K_{W}(p)$ is at most $p$-valent follows from the characterization $K_{W B}(p)$. By the integral (8), $F^{\prime}$ has exactly the same number of zeros as $g$. Since $g$ may have fewer than $p-1$ zeros when $p>1, F$ need not be in $K(p)$.

In order to show that $K_{W E}(p) \subseteq K_{W O}(p)$ we prove a couple of lemmas.

Lemma 1. Let

$$
g(z, t)=\frac{1+e^{-i t} z}{1-e^{-i t} z} \cdot \prod_{i=1}^{n} \Psi\left(z, z_{i}\right)
$$

where $\left|z_{i}\right| \leq 1,1 \leq i \leq n, z \in \mathbf{C}$ and $t \in[0,2 \pi]$. Then $\liminf { }_{r \rightarrow 1-}[\min \operatorname{Re} g(z, t)] \geq$ 0 where the minimum is taken over all $z \in C_{r}$ and all $t \in[0,2 \pi]$.

Proof. For $t$ fixed, $g(z, t)$ is continuous on $C_{1}$, except possibly at $z=e^{i t}$, and pure imaginary on $C_{1}$. If $g(z, t)$ is continuous on $C_{1}$, then

$$
\lim _{r \rightarrow 1-}\left[\min _{\lfloor z \mid=r} \operatorname{Re} g(z, t)\right]=0 \text {. }
$$

If $g(z, t)$ has a pole at $z=e^{i t}$, then it has a pole of order one, so that $g(z, t)$ is conformal in some disc $D$ centered on $e^{i t}$. Since $g(z, t)$ is pure imaginary on $C_{1}$, either $\operatorname{Re} g(z, t)$ is positive for all $z \in D \cap U$ or $\operatorname{Re} g(z, t)$ is negative for all $z \in D \cap U$. Since $g(z, t)$ has a pole at $z=e^{i t}, z_{i} \neq e^{i t}, 1 \leq i \leq n$. It is easily seen that for $z=r e^{i t}, z \in D$ and $\operatorname{Re} g(z, t)>0$ provided $r$, which is less than 1 , is sufficiently near 1 . Therefore, $\operatorname{Re} g(z, t)>0$ for all $z \in D \cap U$.

In any case, for $t$ fixed,

$$
\liminf _{r \rightarrow 1-}\left[\min _{z \mid=r} \operatorname{Re} g(z, t)\right] \geq 0
$$

For each positive real number $\epsilon$, let

$$
r(t)=r(t, \epsilon)=\inf \left\{r: \operatorname{Reg}\left(r e^{i \theta}, t\right)>-\epsilon \text { for all } \theta\right\} .
$$

Let $\epsilon_{1}>0$ be given with $r(t)+\epsilon_{1}<1$. If we choose $r$ such that $r(t)<r<$ $r(t)+\epsilon_{1}$, then $\operatorname{Re} g\left(r e^{i \theta}, t\right)>-\epsilon+\alpha$ for all $\theta$, where $\alpha$ is some positive number. Since $g$ is continuous in $t, \operatorname{Re} g\left(r e^{i \theta}, t^{\prime}\right)>-\epsilon$ for all $\theta$ in $[0,2 \pi]$ and all $t^{\prime}$ in some neighborhood of $t$. Therefore $r\left(t^{\prime}\right)<r(t)+\epsilon_{1}$. This means, by definition, $r(t)$ is upper-semicontinuous on $[0,2 \pi]$. But any such function takes its maximum. That is, there is a $t_{0} \in[0,2 \pi]$ such that $r(t) \leq r\left(t_{0}\right)<1$ for all $t \in[0,2 \pi]$. 
Therefore $\inf \operatorname{Re} g(z, t) \geq-\epsilon$ where the infimum is taken over all $z$ with $r\left(t_{0}\right)<$ $|z|<1$, and $t \in[0,2 \pi]$. This completes the proof of Lemma 1 .

Lemma 2. Suppose that $f$ is regular in $U$ and that $\operatorname{Re} f(z)>0$ for all $z \in U$. Then

$$
\liminf _{r \rightarrow 1-}\left[\min _{|z|=r} \operatorname{Re}\left\{f(z) \prod_{i=1}^{n} \Psi\left(z, z_{i}\right)\right\}\right] \geq 0
$$

whenever $\left|z_{i}\right| \leq 1$ for $1 \leq i \leq n$.

Proof. Assume that $f(0)=1$. With this normalization we can apply the Herglotz representation theorem for functions with positive real part:

There is an increasing function $\alpha:[0,2 \pi] \rightarrow[0,1]$ such that $\alpha(0)=0$, $\alpha(2 \pi)=1$, and

$$
f(z)=\int_{0}^{2 \pi} \frac{1+e^{-i t} z}{1-e^{-i t} z} d \alpha(t)
$$

See, for instance, Wall [6, p. 275]. Therefore,

$$
\operatorname{Re} f(z) \prod \Psi\left(z, z_{i}\right)=\int_{0}^{2 \pi} \operatorname{Re}\left\{\frac{1+e^{-i t} z}{1-e^{-i t} z} \prod \Psi\left(z, z_{i}\right)\right\} d \alpha(t) .
$$

Let $\epsilon>0$ be given. By Lemma 1 , there is an $r_{\epsilon}<1$ such that

$$
\operatorname{Re}\left\{\frac{1+e^{-i t} z}{1-e^{-i t} z} \prod \Psi\left(z, z_{i}\right)\right\}>-\epsilon
$$

whenever $|z|>r_{\epsilon}$. Thus, for $|z|>r_{\epsilon}$,

$$
\operatorname{Re} f(z) \prod \Psi\left(z, z_{i}\right)>\int_{0}^{2 \pi}-\epsilon d \alpha(t)=-\epsilon .
$$

This proves Lemma 2 for the case $f(0)=1$.

Suppose $f(0)=a+i b$. Then $a>0$, and $g(z)=(f(z)-i b) / a$ is regular in $U$ with $g(0)=1$ and has positive real part. Since $f(z) \Pi \Psi\left(z, z_{i}\right)=a g(z) \Pi \Psi\left(z, z_{i}\right)+$ $i b \Pi \Psi\left(z, z_{i}\right)$ and $\lim _{r \rightarrow 1}-\operatorname{Re} i b \Pi \Psi\left(z, z_{i}\right)=0$, Lemma 2 is seen to be correct in any case.

Proposition 8. $K_{W E}(p) \subseteq K_{W O}(p)$.

Proof. Let $F \in K_{W E}(p)$. Then there is a function $f \in S_{w c}(p)$ such that $f(0)=$ 0 and $\operatorname{Re}\left\{z F^{\prime}(z) / f(z)\right\}>0$ for all $z \in U$. Let $f(z)=[b(z)]^{p} \Pi_{i=1}^{p-1} \Psi\left(z, z_{i}\right)$. Now

$$
\begin{aligned}
\underset{r \rightarrow 1-}{\liminf }\left[\min _{\lfloor z \mid=r} \operatorname{Re}\left\{\frac{z F^{\prime}(z)}{[b(z)]^{p}}\right\}\right] \\
\quad=\liminf _{r \rightarrow 1-}\left[\min _{\lfloor z \mid=r} \operatorname{Re}\left\{\left(\frac{z F^{\prime}(z)}{f(z)}\right) \prod \Psi\left(z, z_{i}\right)\right\}\right] \geq 0 \text { by Lemma } 2 .
\end{aligned}
$$


Therefore $F \in K_{W O}(p)$. This completes the proof of Proposition 8 and thus also the proof of Theorem 1 .

\section{REFERENCES}

1. J. A. Hummel, Multivalent starlike functions, J. Analyse Math. 18 (1967), 133-160. MR $35 \# 359$.

2. W. Kaplan, Close-to-convex schlicht functions, Michigan Math. J. 1 (1952), 169185. MR 14, 966 .

3. A. E. Livingston, p-valent close-to-convex functions, Trans. Amer. Math. Soc. 115 (1965), 161-179. MR $33 \# 7520$.

4. D. Styer, On weakly starlike multivalent functions, J. Analyse Math. (to appear).

5. T. Umezawa, On the theory of univalent functions, Tôhoku Math. J. (2) 7 (1955), 212-228. MR 17, 1068.

6. H. S. Wall, Analytic theory of continued fractions, Chelsea, New York, 1967.

DEPARTMENT OF MATHEMATICS, UNIVERSITY OF CINCINNATI, CINCINNATI, OHIO 45221 